\title{
Identification of intrinsic and reflexive contributions to trunk stabilization in patients with low back pain: a case-control study
}

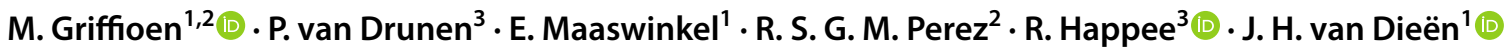

Received: 15 October 2019 / Accepted: 18 March 2020 / Published online: 2 April 2020

(c) The Author(s) 2020

\begin{abstract}
Purpose The goal of this study was to assess differences in low back stabilization and underlying mechanisms between patients with low back pain (LBP) and healthy controls. It has been hypothesized that inadequate trunk stabilization could contribute to LBP through high tissue strains and/or impingement. Evidence to support this is inconsistent, and not all methods that have been used to study trunk stabilization are equally suitable. We have recently developed a method to assess intrinsic and reflexive contributions to trunk stabilization, which aims to circumvent the limitations of previous studies.

Methods Forty-nine participants suffering from chronic LBP and a control group of fifty healthy subjects participated in this study. Trunk stabilization was measured using force-controlled perturbations directly applied to the trunk. The actuator displacement and contact force between the actuator and subject were measured as well as electromyography (EMG) of the M. Longissimus. Underlying mechanisms were characterized using system identification.

Results LBP patients showed lower admittance, i.e., less displacement per unit of force applied, mainly due to higher position, velocity and acceleration feedback gains. Among patients, lower trunk admittance and higher reflex gains were associated with more negative pain-related cognitions.

Conclusion Trunk stabilization differs between LBP patients and controls, with the same perturbations causing less trunk movement in patients, due to stronger reflexes. We interpret these changes as reflecting protective behavior.
\end{abstract}

\section{Graphic abstract}

These slides can be retrieved under Electronic Supplementary Material.
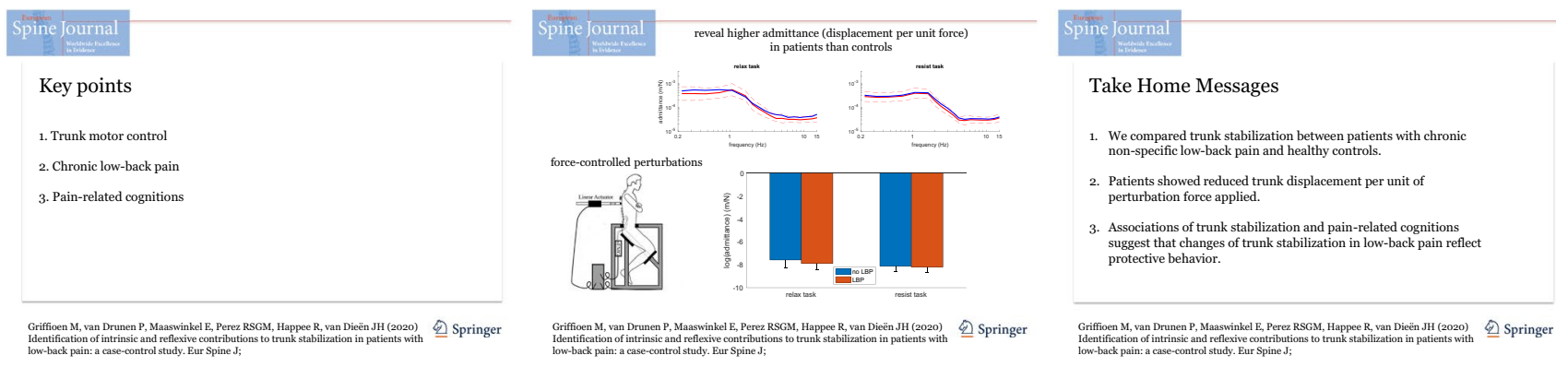

Keywords Low back pain $\cdot$ Stability $\cdot$ Reflex $\cdot$ Stiffness $\cdot$ Pain-related cognitions

Electronic supplementary material The online version of this article (https://doi.org/10.1007/s00586-020-06385-9) contains supplementary material, which is available to authorized users.

\section{J. H. van Dieën}

J.van.Dieen@VU.nl

Extended author information available on the last page of the article

\section{Introduction}

Inadequate trunk stabilization has been hypothesized to contribute to low back pain (LBP) [1]. Trunk stabilization is often assessed by studying responses to mechanical perturbations. Using this approach, several studies have shown longer response delays after external mechanical 
perturbations of trunk posture in LBP patients than in controls [2], which has been interpreted as an impairment and a cause of the incidence and persistence of LBP [3-5]. In apparent contrast to impaired stabilization, higher trunk stiffness, i.e., a higher mechanical resistance to perturbations, has been reported [6], possibly due to altered trunk muscle recruitment patterns in patients compared to controls [7]. These changes have been interpreted as adaptive responses to enhance control over trunk movement and therewith prevent pain provocation [7]. In fact, increased trunk muscle activity and increased trunk stiffness could explain the longer response delays found in LBP patients. Higher muscle activity before the perturbation would render detection of reflex onsets, i.e., the onset of a change in muscle activity after the perturbation, more difficult causing overestimated delays. In addition, with increased stiffness, the same mechanical disturbance will cause a smaller and slower deviation of trunk posture. Consequently, the perturbation might be sensed later, causing a later change in excitation of trunk muscles. So paradoxically, the finding of increased response delays might not reflect a slower reflex loop, but a methodological issue or a change in the input into the reflex loop resulting from functional, adaptive responses to enhance trunk stabilization.

We have recently developed a method to assess intrinsic and reflexive contributions to trunk stabilization, which aims to circumvent the limitations described above [8-10]. The method was shown to have good reliability in healthy subjects and LBP patients [9]. The present study used this method to assess differences in trunk stabilization between LBP patients and healthy controls when maximally resist the perturbation or when sitting relaxed but upright, to investigate their maximal ability, natural behavior and their ability to modulate between both tasks. Based on findings of changed muscle recruitment [7] and intrinsic stiffness [6], we hypothesized that LBP patients would show lower admittance during the relax task (explained by higher intrinsic stiffness and damping) and, related to that, less modulation between the relax task and resist task. Given the methodological issues regarding onset determination in previous studies, we expected no difference in reflex delays between the groups.

\section{Materials and methods}

\section{Participants}

Forty-nine participants suffering from LBP and a control group of fifty healthy subjects participated in this study. The groups were group matched on sex and height, but the patient group was significantly older and heavier (Table 1). The patients suffered from nonspecific LBP for at least
Table 1 Information about the patient and control group

\begin{tabular}{|c|c|c|c|c|}
\hline & Patients & Controls & t statistic & $P$ value \\
\hline $\begin{array}{l}\text { Gender } \\
\quad(\# \text { male/\#female })\end{array}$ & $28 / 21$ & $27 / 23$ & - & - \\
\hline Age (years) & $48 \pm 10$ & $35 \pm 16$ & -3.75 & $<0.001^{*}$ \\
\hline Body length (cm) & $176 \pm 10$ & $175 \pm 09$ & -0.49 & 0.653 \\
\hline Body weight (kg) & $83 \pm 19$ & $71 \pm 13$ & -3.53 & $<0.001 *$ \\
\hline Momentary pain (-) & $4.6[0.3-9]$ & - & & \\
\hline Pain diaries $[-]$ & $6.2 \pm 1.5$ & - & & \\
\hline $\begin{array}{l}\text { Absence current year } \\
\text { (days) }\end{array}$ & 196 [90-365] & - & & \\
\hline Oswestry LBPQ [-] & $37 \pm 18$ & - & & \\
\hline Back beliefs [-] & $28 \pm 7$ & - & & \\
\hline Tampa scale [-] & $41 \pm 10$ & - & & \\
\hline HADS [-] & $13[2-37]$ & - & & \\
\hline PCS [-] & $20[2-52]$ & - & & \\
\hline
\end{tabular}

Average values are given with their standard deviations $( \pm)$ or range ([xxx])

*Significant difference

3 months and were under treatment by physical therapists (9), pain specialists (26) and rehabilitation centers (14). The healthy participants had not experienced LBP in the 12 months prior to the experiments. None of the participants suffered from radicular pain or other (neurological) disorders or used medication that could affect balance control. All participants gave informed consent according to the guidelines of the medical ethical committee of VU Medical Center, Amsterdam (2013/37).

Throughout the measurements, patients reported momentary pain six times with a BS-11 score [11]. In the week prior to the measurement, they filled in a pain diary for seven days, containing BS-11 scores in the morning, afternoon, evening, and scoring the lowest and highest pain level during the day. The LBP patients also filled in the questionnaires on illness beliefs, fear of movement, catastrophizing, depression and anxiety: the Oswestry Disability Index (ODI) [12], Back Beliefs Questionnaire (BBQ) [13], Tampa Scale for Kinesiophobia (TSK) [14], Hospital Anxiety and Depression Scale (HADS) [15] and Pain Catastrophizing Scale (PCS) [16]. Averages of their scores are found in Table 1. In view of high correlations between questionnaire scores, two composite scores were constructed. A pain and disability score was calculated as the average of z-normalized momentary pain scores, pain diary scores and ODI scores, and a pain-related cognition score was calculated as the average of z-normalized scores on the BBQ, TSK, HADS and PCS.

\section{Measurements}

In the experimental setup, subjects were seated in a kneeling-seated posture with their pelvis restrained (Fig. 1). 


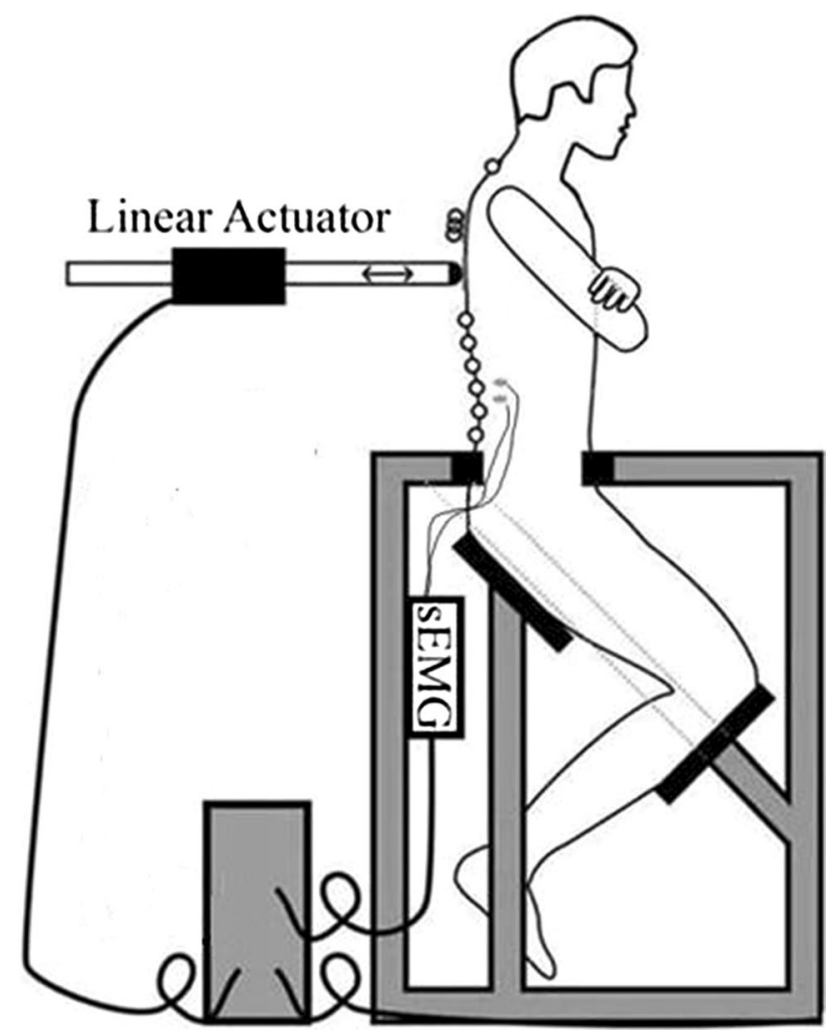

Fig. 1 Experimental setup

Force perturbations were applied in ventral direction on the T10 spinous process by a magnetically driven linear actuator (Servotube STB2510S Forcer and Thrustrod TRB25-1380, Copley Controls, MA, USA), with a thermoplastic patch $(4 \times 4 \mathrm{~cm})$ placed between the subject and the actuator to improve force transfer and comfort. During the trials, subjects were instructed to keep their eyes closed, arms crossed in front of the chest, and to minimize lateral excursions. Task instructions were to 'maximally resist the perturbation' by minimizing flexion/extension excursions (resist task), or to 'relax but remain sitting upright' (relax task).

Each trial had a duration of $50 \mathrm{~s}$ and consisted of a linearly increasing force ramp of $3 \mathrm{~s}$ from $0 \mathrm{~N}$ to $60 \mathrm{~N}$ followed by a crested multi-sine signal [17] with a $35 \mathrm{~N}$ amplitude superimposed on a $60 \mathrm{~N}$ baseline preload.

The actuator displacement and contact force between the actuator and subject were measured, as well as surface electromyography (EMG) of the lumbar part of the bilateral M. Longissimus at level L3 and L4 recorded at 2048 samples/s (REFA, TMSi, the Netherlands). The M. Longissimus was chosen because of the high coherence between this muscle's activity and thorax displacement [8].

\section{System identification}

Closed loop identification techniques [17] were used to describe the translational low back admittance and reflexes as frequency response functions (FRFs). The admittance FRF describes the actuator displacement as a function of the contact force, representing the resistance against the perturbation as a function of frequency. The reflex FRF describes the EMG amplitude of the lumbar part of the M. Longissimus as a function of the actuator displacement.

Coherences of admittance and reflex FRFs were assessed for the frequencies containing power in the perturbation signal. Coherence ranges from zero to one, where one reflects a perfect, noise-free relation between input and output. A coherence greater than 0.24 was considered significant with $\alpha \leq 0.05$ [18].

Because task-related modulation of the FRFs mainly occurs below the natural frequency around $1.1 \mathrm{~Hz}$, and because trunk mass dominates responses at higher frequencies [8], low-frequency gains (LF gains) of the admittance and reflexive FRFs were defined by averaging over the five frequency pairs within the $0.2-1.1 \mathrm{~Hz}$ range.

\section{Parametric identification}

A linear neuromuscular control model was constructed to translate the FRFs into physiological elements representing intrinsic and reflexive contributions $[8,10]$. Model parameters were estimated by optimizing the fit of the model transfer functions to the transfer functions estimated as described above, using simulated annealing. The effective trunk mass $(m)$ was fitted. The stabilizing properties of passive tissues and muscle co-contraction and the destabilizing effect of trunk mass were lumped into two parameters describing the overall intrinsic stiffness and damping $(k, b)$ of the low back. The reflexive contribution was described by position, velocity and acceleration feedback gains $\left(k_{p}, k_{v}, k_{a}\right)$ with a single reflex delay $\left(\tau_{\text {ref }}\right)$. Muscle activation dynamics were implemented as a second-order system [19] with a cutoff frequency and a dimensionless damping, set to $0.75 \mathrm{~Hz}$ and 1.05 , respectively, as the average activation dynamics in van Drunen et al. [8]. Contact dynamics between the subjects' trunk and the actuator were estimated as a spring and damper $\left(k_{c}, b_{c}\right)$.

The validity of the optimized model and its parameters was assessed in the time domain using the variance accounted for $(V A F)$. A $V A F$ of $100 \%$ reflects a perfect description of the measured signal by the model at the input frequencies.

For more detailed information on the perturbation characteristics, data recording, data processing, system identification and parametric identification, see van Drunen et al. [8] and van Dieën et al. [10]. 

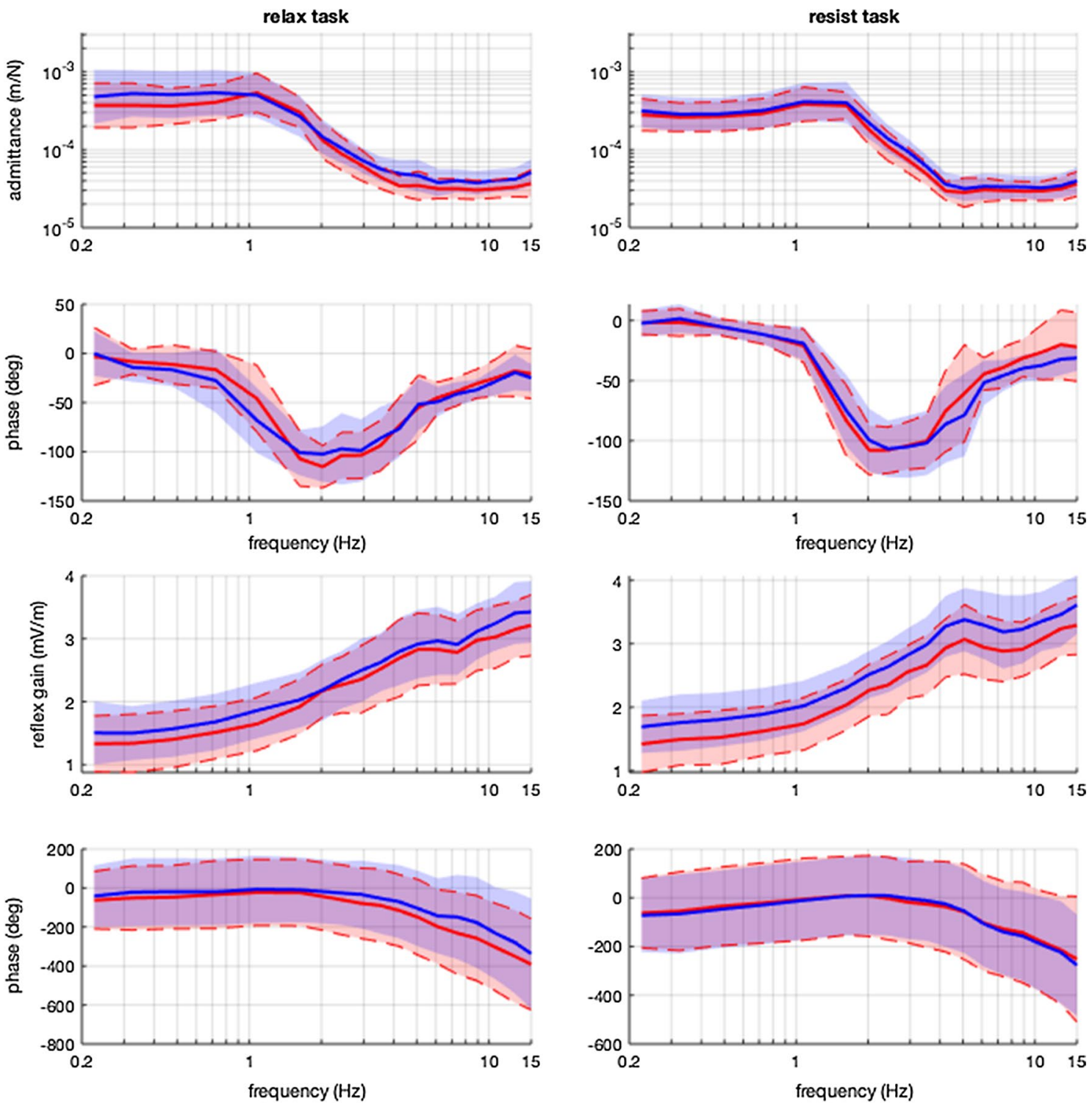

Fig. 2 Group-averaged frequency response functions (FRFs) of the admittance (upper two rows) and EMG (lower two rows). Results for controls (blue lines) and patients (red lines) during the relax task (left) and resist task (right) were averaged over subjects (with shad-

\section{Statistics}

The FRF gains and LF gains were log-transformed to satisfy the assumption of normality. To test our hypotheses, nonparametric LF gains and parametric parameters were compared between tasks and groups with generalized estimating equations (GEE) with LBP, task, task*LBP, age and LBP*age as predictors. In this analysis, age was dichotomized based on the median age of all participants. Preliminary checks were conducted to ensure validity of the assumption of normality, linearity, homogeneity of variances and homogeneity of regression slopes. Body mass was not used as a covariate,

ows as standard deviation). The gain (amplitude difference) and phase (time shift) illustrate the transformation of the input signal into the output signal

since effective mass is accounted for in the parametric estimation.

Linear correlation analyses were performed to test the relation between trunk stabilization parameters, pain and disability and pain-related cognitions. Pearson's R ranges from $0-1$ where $<0.3$ indicates a 'poor' correlation, $0.3-0.5$ represents a 'moderate' correlation and $>0.5$ represents a 'strong' correlation [20].

For all tests, results with $p$-values smaller than 0.05 (twosided) were considered significant. Processing, system identification, parametric identification and statistical analyses of the data were performed using MATLAB (The Mathworks, Inc., Natick, MA, USA). 

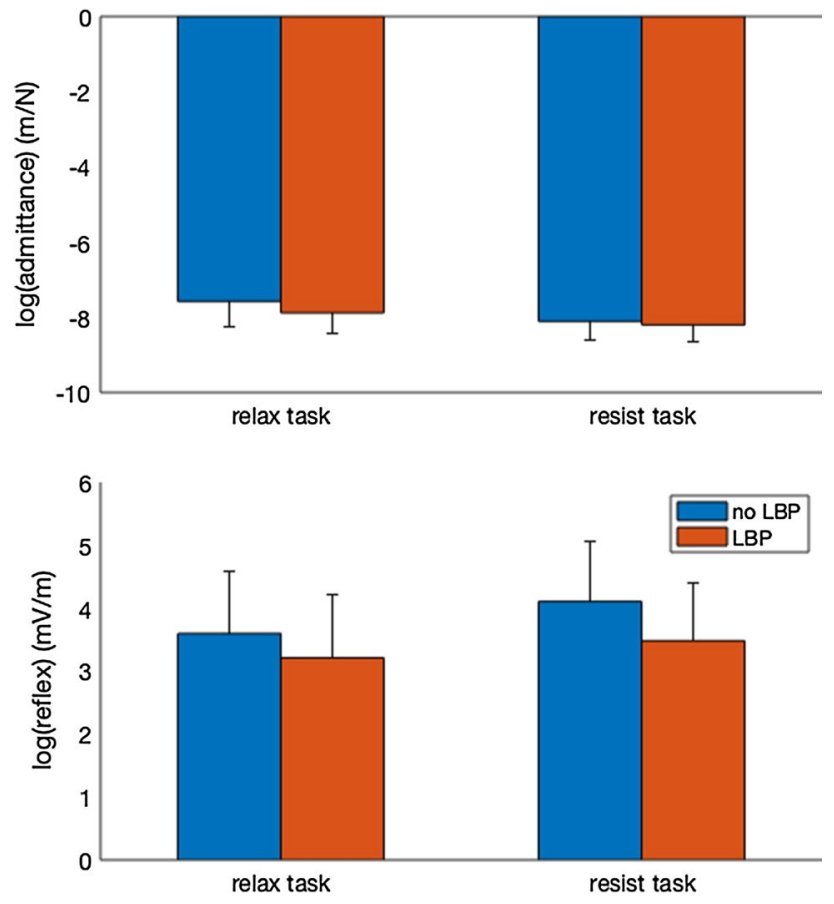

Fig. 3 Box plots for admittance (top window) and reflexes (bottom window) showing lower admittance and lower reflexes in patients (red bars) compared to healthy controls (blue bars). Error bars represent one standard deviation. * denotes significance at the $p<0.05$ level

\section{Results}

\section{Group-level comparison between LBP patients and controls}

Mean admittance coherence was high (average and standard deviation: $0.93 \pm 0.05$ ), indicating good input-output correlations, while the mean EMG coherence $(0.73 \pm 0.10)$ was good considering the noisy character of EMG signals.

A significant main effect of LBP was found on LF admittance, with as hypothesized, lower admittance in LBP patients than in healthy subjects (Figs. 2, 3 and Table 2). In contrast to our hypotheses, damping and stiffness did not differ between groups (Fig. 4). Position, velocity and acceleration reflex gains, however, were significantly higher in LBP patients. The hypothesized larger modulation in healthy controls compared to LBP patients (task*LBP interaction) was only seen in velocity reflex gains, and a trend was seen in admittance. As expected, there was no significant difference in reflex delays between LBP patients and healthy controls.

Task and age effects were found in LF admittance gains (higher in the relax condition and younger subjects). Also, task effects were found for LF reflex gains, acceleration and velocity reflex gains (lower in the relax condition). Interaction effects between LBP and age were found on LF admittance gains, damping and position reflex gains, showing smaller differences between LBP patients and healthy controls in the older group. Effective mass was larger in participants with LBP, in line with their larger body mass.

Histograms of the LF admittance gains (Fig. 5) illustrate the difference in admittance between groups, but also show large overlap between LBP patients and healthy controls in both conditions and do not suggest subgroups among LBP patients.

\section{Relation of trunk stabilization with pain and disability, and cognition}

LF admittance gain in the relax condition was moderately correlated with pain and disability, but also with pain-related cognitions (Table 3). Moderate correlations of intrinsic damping and velocity reflex gains in both conditions and of

Table 2 B-values of the effects of LBP, age, task, the interaction effect of task and LBP and the interaction effect of age and LBP

\begin{tabular}{|c|c|c|c|c|c|c|c|c|c|c|}
\hline & \multicolumn{2}{|l|}{ LBP } & \multicolumn{2}{|l|}{ Age } & \multicolumn{2}{|l|}{ Task } & \multicolumn{2}{|c|}{ Task*LBP } & \multicolumn{2}{|c|}{ Age*LBP } \\
\hline & $B$ value & $P$ value & B value & $P$ value & $B$ value & $P$ value & $B$ value & $P$ value & $B$ value & $P$ value \\
\hline $\mathrm{LF}$ admittance $(\mathrm{m} / \mathrm{N})$ & -0.45 & $<0.000^{*}$ & -0.60 & $<0.000^{*}$ & -0.53 & $<0.000^{*}$ & 0.21 & 0.138 & 0.55 & $<0.000^{*}$ \\
\hline $\mathrm{LF}$ reflex $(\mathrm{mV} / \mathrm{m})$ & -0.23 & 0.326 & 0.80 & $<0000^{*}$ & 0.51 & $0.006^{*}$ & -0.24 & 0.363 & -0.67 & $0.017 *$ \\
\hline B (Ns/m) & 0.45 & 0.099 & 0.63 & $0.007 *$ & -0.18 & 0.395 & 0.27 & 0.377 & -1.00 & $0.002 *$ \\
\hline $\mathrm{K}(\mathrm{N} / \mathrm{m})$ & -0.45 & 0.203 & 0.69 & $0.021 *$ & -0.30 & 0,271 & 0.51 & 0.191 & -0.03 & 0.935 \\
\hline $\mathrm{bc}(\mathrm{Ns} / \mathrm{m})$ & 29.9 & 0.412 & 52.6 & 0.159 & - & & - & & -45.6 & 0.385 \\
\hline $\mathrm{kc}(\mathrm{N} / \mathrm{m})$ & 0.43 & $0.013^{*}$ & 0.77 & $<0.000^{*}$ & - & & - & & -0.79 & $0.001 *$ \\
\hline $\mathrm{Ka}\left(\mathrm{Ns}^{2} / \mathrm{m}\right)$ & 0.25 & $0.049^{*}$ & -0.16 & 0.136 & 0.33 & $<0.000 *$ & -0.23 & 0.101 & 0.03 & 0.824 \\
\hline $\mathrm{Kv}(\mathrm{Ns} / \mathrm{m})$ & 1.91 & $<0.000^{*}$ & -0.32 & 0.446 & 1.54 & $<0.000^{*}$ & -1.27 & $0.019 *$ & -0.66 & 0.258 \\
\hline $\mathrm{Kp}(\mathrm{N} / \mathrm{m})$ & 0.99 & $0.011^{*}$ & 0.35 & 0.287 & 0.55 & 0.068 & -0.28 & 0.512 & -1.19 & $0.010^{*}$ \\
\hline Reflex delay (s) & -0.00 & 0.640 & -0.00 & 0.429 & - & & - & & 0.01 & 0.210 \\
\hline
\end{tabular}

Age was dichotomized on the median of 43 years

*Significant difference 

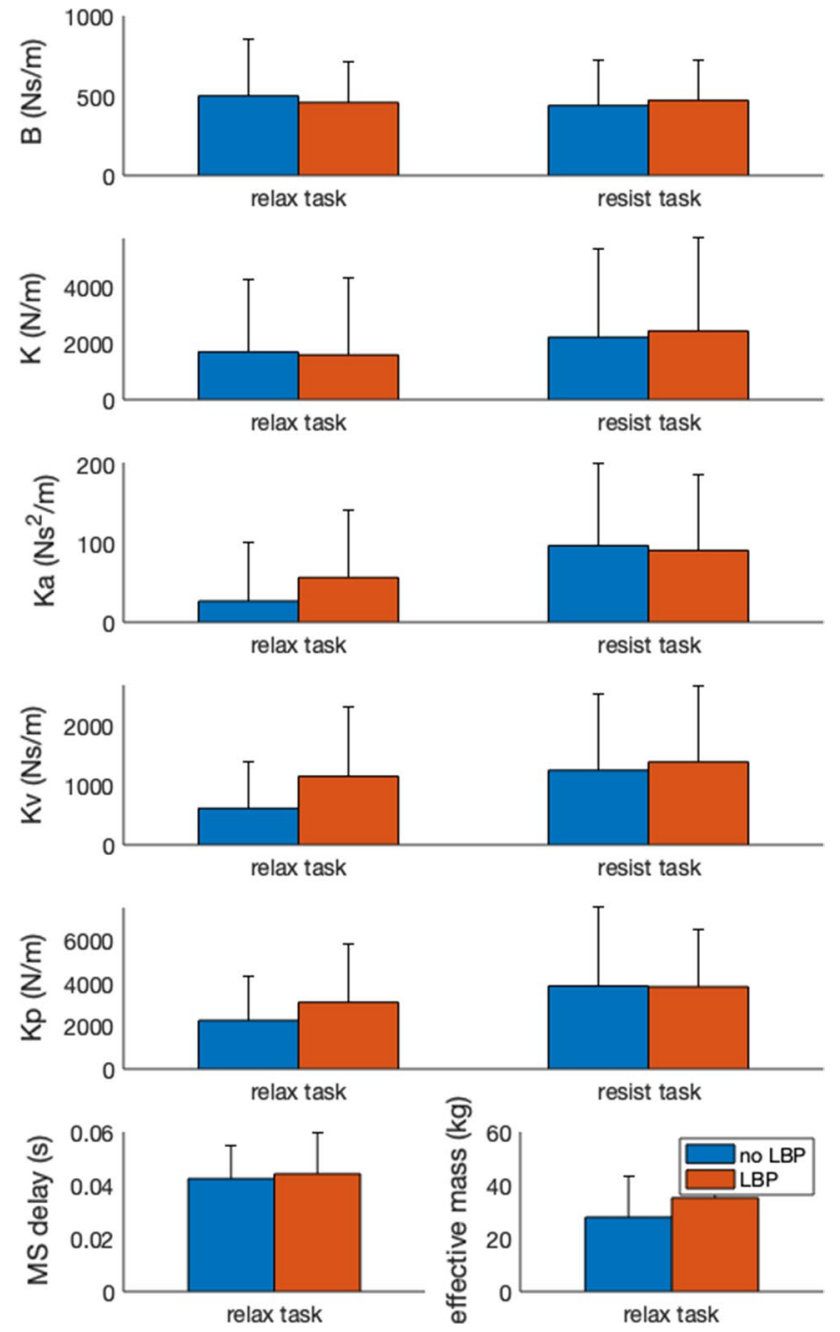

Fig. 4 Box plots for the parametric parameters for the relax task (left window) and the resist task (right window) showing differences between patients (red bars) and healthy controls (blue bars). Error bars represent one standard deviation. *denotes significance at the $p<0.05$ level

position feedback gains in the relax condition were found with pain-related cognitions only.

\section{Discussion}

We used a novel system identification technique to assess differences in mechanisms of low back stabilization between LBP patients and healthy controls. We hypothesized that LBP patients would show lower admittance during the relax task, because of higher intrinsic stiffness and damping, and less modulation between the relax and resist tasks. In the relax task, we found the expected lower admittance in patients with LBP, and this effect was also found in the resist task. The group differences were larger during the relax task than during the resist task, but modulation between tasks was not significantly different between groups. As expected, parametric identification showed no effects on reflex delays and additionally showed that differences in trunk stabilization between LBP patients and healthy subjects were mostly due to increased position, velocity and acceleration feedback gains in LBP patients. So, in contrast to our hypothesis, differences in trunk stabilization between LBP patients and controls were not dependent on intrinsic stiffness and damping and hence not on feed forward motor control changes, but on enhanced feedback control.

Johansson and Sojka [21] introduced a pathophysiological model that might explain the increase in feedback gains found in our study. The model describes how nociception activates gamma motor neurons and consequently enhances muscle spindle feedback. However, in the present study we found no correlation between pain intensity as rated with the pain and disability questionnaires and reflex gains. Alternatively, the increased reflex gains in LBP patients could be a pain-induced behavioral change toward a more protective or more conservative behavior [22]. This interpretation is consistent with the associations found between outcomes reflecting trunk stabilization and pain-related cognitions, whereas correlations with pain and disability were less strong. Fear of movement, illness beliefs, catastrophizing and depression results coincided with a tighter control over trunk posture in LBP patients. This is in line previously reported associations of trunk stiffness with fear of movement [23] and of the level of trunk muscle activity during walking and catastrophizing [24] in chronic LBP patients. Osumi et al. [25] also have recently published a study on the effect of kinesiophobia on lumbar movements. They divided their LBP group in a 'high-fear' and 'low-fear' group based on their TSK scores and found that in voluntary lumbar bending movements, the high-fear group took longer on movement initiation and during the switch in movement direction. No differences were found between the low-fear group and healthy controls, also pointing toward a fear-based protective tighter control other than one that results from pain. We found no evidence to suggest subgroups among LBP patients with 'tight' and 'loose' control as proposed by van Dieen et al. [26]. One might expect 'loose' control to be most prominent during the relax task, but instead, we observed a lower admittance in the LBP group in both conditions. It should be taken in account that our method involves a $60 \mathrm{~N}$ baseline preload, which might make the relax task too demanding to allow 'loose' control.

The age effect and age*LBP interaction effect on admittance found in this study emphasize the need to take age in account when analyzing trunk stabilization. We decided to dichotomize age in our analysis because the effect of age is 
Fig. 5 Histograms of LF admittance gains of LBP patients and healthy controls in the relax task (top window) and resist task (bottom window) showing lower values for patients on average, but with substantial overlap between groups. The blue bars represent the controls, the red bars represent the LBP patients, and the purple color represents the overlap between the two groups
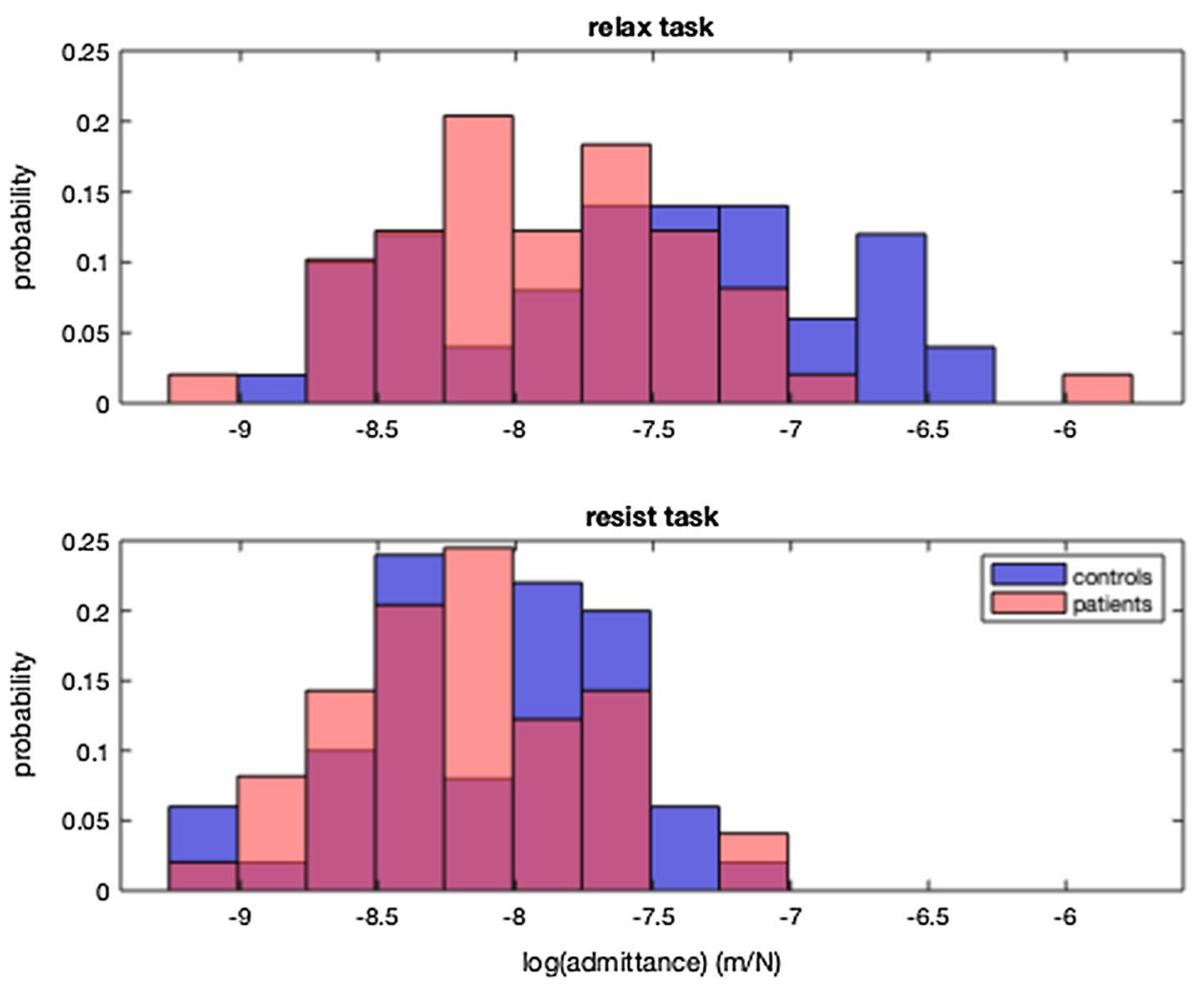

Table 3 Correlations between trunk stability parameters and pain and disability and cognitions

\begin{tabular}{|c|c|c|c|c|}
\hline & \multicolumn{2}{|c|}{ Pain and disability } & \multicolumn{2}{|l|}{ Cognitions } \\
\hline & Pearson's R & $P$ value & Pearson's R & $P$ value \\
\hline $\begin{array}{l}\text { LF admittance relax } \\
\qquad(\mathrm{m} / \mathrm{N})\end{array}$ & -0.30 & $0.037 *$ & -0.31 & $0.027 *$ \\
\hline $\begin{array}{l}\text { LF admittance resist } \\
(\mathrm{m} / \mathrm{N})\end{array}$ & -0.05 & 0.709 & -0.15 & 0.306 \\
\hline LF reflex relax $(\mathrm{mV} / \mathrm{m})$ & 0.16 & 0.262 & 0.14 & 0.325 \\
\hline $\mathrm{LF}$ reflex resist $(\mathrm{mV} / \mathrm{m})$ & 0.20 & 0.164 & 0.08 & 0.572 \\
\hline B relax (Ns/m) & 0.16 & 0.271 & 0.34 & $0.017 *$ \\
\hline B resist $(\mathrm{Ns} / \mathrm{m})$ & 0.12 & 0.384 & 0.33 & $0.022^{*}$ \\
\hline $\mathrm{K}$ relax $(\mathrm{N} / \mathrm{m})$ & 0.01 & 0.957 & -0.03 & 0.862 \\
\hline $\mathrm{K}$ resist $(\mathrm{N} / \mathrm{m})$ & -0.11 & 0.459 & -0.13 & 0.378 \\
\hline Ka relax $\left(\mathrm{Ns}^{2} / \mathrm{m}\right)$ & 0.19 & 0.193 & 0.20 & 0.162 \\
\hline Ka resist $\left(\mathrm{Ns}^{2} / \mathrm{m}\right)$ & 0.15 & 0.308 & 0.10 & 0.487 \\
\hline Kv relax (Ns/m) & 0.24 & 0.102 & 0.31 & $0.032 *$ \\
\hline $\mathrm{Kv}$ resist (Ns/m) & 0.22 & 0.133 & 0.31 & $0.033^{*}$ \\
\hline Kp relax (N/m) & 0.14 & 0.334 & 0.29 & $0.045^{*}$ \\
\hline Kp resist $(\mathrm{N} / \mathrm{m})$ & 0.04 & 0.804 & 0.09 & 0.533 \\
\hline Reflex delay (s) & 0.08 & 0.576 & -0.19 & 0.194 \\
\hline
\end{tabular}

*Significant correlations

not necessarily linear, and in this way the interaction is easier to interpret. Because dichotomization holds the chance of misclassification, we also performed an analysis without dichotomization. This did not result in different conclusions.
Some limitations of this study should be considered when interpreting the results. We only measured EMG from the M. Longissimus as most representative for the muscle responses to the imposed perturbations, where other muscles including deep muscles, which would require intramuscular electromyography recordings, could hold independent information regarding trunk stabilization. Also, we only collected data from subjects experiencing small sagittal plane perturbations of an upright position and therefore will not have captured all relevant aspects of trunk motor control [27, 28]. Finally, most patients will have had physiotherapy, which often includes some form of motor control exercise (often stabilization exercise), and this may have contributed to the lower admittance. However, stabilization exercise does not specifically address reflex control and this was found to be the main factor underlying differences in impedance. Also treatment effects would not offer a full explanation for decreased admittance during the 'relax task.'

As mentioned above, we interpret the difference between healthy subjects and LBP patients as an adaptation to protect the lower back. However, it is questionable whether this adaptive behavior is of benefit in the long term. Van Dieën et al. [22] suggested that in the long term more rigid control of posture and movement can be harmful, because the related muscle activity causes high compressive loading on the spine, which may contribute to injury and degeneration, and it reduces variability of the afferent signals from the lumbar area to the central nervous system, which could cause neuroplastic changes that 
impair motor control. Combined with the motor control differences found in the present study, this would suggest that motor control exercises, at least in patients with low admittance, should aim for increasing admittance, inhibition of lumbar muscle activity and reduction in adverse pain-related cognitions.

In conclusion, LBP patients showed lower admittance during trunk stabilization tasks based on higher position, velocity and acceleration feedback gains. Furthermore, our results indicate a relation between low admittance and adverse cognitions regarding pain in LBP patients. Overall, these results support interpretation of changes in trunk motor control in LBP as protective behavior.

Acknowledgements This study was supported by the Dutch Technology Foundation STW, which is part of the Netherlands Organization for Scientific Research (NWO) and which is partly funded by the Ministry of Economic Affairs. See www.neurosipe.nl-Project 10732: QDISC.

Funding Leila Alizadehsaravi was funded by the European Union's Horizon 2020 research and innovation programme under the Marie Skłodowska-Curie grant agreement No 721577. Sjoerd Bruijn was funded by a VIDI grant (016.Vidi.178.014) from the Dutch Organization for Scientific Research (NWO).

\section{Compliance with ethical standards}

Conflict of interest The authors report no other conflicts of interest.

Ethical approval All procedures in this study were in accordance with the ethical standards of the institutional research committee and with the 1964 Helsinki Declaration and its later amendments and comparable ethical standards. The procedures were approved by the ethical review board of the Faculty of Behavioral \& Movement Sciences, VU Amsterdam (VCWE-2018-038).

Informed consent All participants provided written informed consent before participation.

Open Access This article is licensed under a Creative Commons Attribution 4.0 International License, which permits use, sharing, adaptation, distribution and reproduction in any medium or format, as long as you give appropriate credit to the original author(s) and the source, provide a link to the Creative Commons licence, and indicate if changes were made. The images or other third party material in this article are included in the article's Creative Commons licence, unless indicated otherwise in a credit line to the material. If material is not included in the article's Creative Commons licence and your intended use is not permitted by statutory regulation or exceeds the permitted use, you will need to obtain permission directly from the copyright holder. To view a copy of this licence, visit http://creativecommons.org/licenses/by/4.0/.

\section{References}

1. Panjabi MM (1992) The stabilizing system of the spine. Part II. Neutral zone and instability hypothesis. J Spinal Dis 5:390-397

2. Prins MR, Griffioen M, Veeger TTJ, Kiers H, Meijer OG, van der Wurff P, Bruijn SM, van Dieen JH (2018) Evidence of splinting in low back pain? a systematic review of perturbation studies. Eur Spine J 27:40-59. https://doi.org/10.1007/s00586-017-5287-0

3. Magnusson ML, Aleksiev AR, Wilder DG, Pope MH, Spratt KF, Lee SH, Goel VK, Weinstein JN (1996) Unexpected load and asymmetric posture as etiologic factors in low back pain. Eur Spine J 5:23-35

4. MacDonald D, Moseley GL, Hodges PW (2010) People with recurrent low back pain respond differently to trunk loading despite remission from symptoms. Spine 35:818-824. https:// doi.org/10.1097/BRS.0b013e3181bc98f1

5. Cholewicki J, Silfies SP, Shah RA, Greene HS, Reeves NP, Alvi K, Goldberg B (2005) Delayed trunk muscle reflex responses increase the risk of low back injuries. Spine 30:2614-2620. https ://doi.org/10.1097/01.brs.0000188273.27463.bc

6. Hodges P, van den Hoorn W, Dawson A, Cholewicki J (2009) Changes in the mechanical properties of the trunk in low back pain may be associated with recurrence. J Biomech 42:61-66. https://doi.org/10.1016/j.jbiomech.2008.10.001

7. van Dieen JH, Selen LP, Cholewicki J (2003) Trunk muscle activation in low-back pain patients, an analysis of the literature. J Electromyogr Kinesiol 13:333-351

8. van Drunen P, Maaswinkel E, van der Helm FC, van Dieen JH, Happee R (2013) Identifying intrinsic and reflexive contributions to low-back stabilization. J Biomech 46:1440-1446. https://doi. org/10.1016/j.jbiomech.2013.03.007

9. Griffioen M, Maaswinkel E, Zuurmond WW, van Dieen JH, Perez RS (2016) Trunk stabilization estimated using pseudorandom force perturbations, a reliability study. J Biomech 49:244-251. https://doi.org/10.1016/j.jbiomech.2015.12.007

10. van Dieen JH, van Drunen P, Happee R (2018) Sensory contributions to stabilization of trunk posture in the sagittal plane. J Biomech 70:219-227. https://doi.org/10.1016/j.jbiom ech.2017.07.016

11. Jensen MP, Karoly P, O’Riordan EF, Bland F, Burns RS (1989) The subjective experience of acute pain an assessment of the utility of 10 indices. Clin J Pain 5:153-160

12. Fairbank JC, Couper J, Davies JB, O'Brien JP (1980) The Oswestry low back pain disability questionnaire. Physiotherapy 66:271-273

13. Symonds TL, Burton AK, Tillotson KM, Main CJ (1996) Do attitudes and beliefs influence work loss due to low back trouble? Occup Med (Oxford, England) 46:25-32. https://doi.org/10.1093/ occmed/46.1.25

14. Kori SH, Miller RP, Todd DD (1990) Kinesiophobia: a new view of chronic pain behavior. Pain Manag 3:35-43

15. Zigmond AS, Snaith RP (1983) The hospital anxiety and depression scale. Acta Psychiatr Scand 67:361-370

16. Sullivan MJL, Bishop SR, Pivik J (1995) The pain catastrophizing scale: development and validation. Psychol Assess 7:524-532

17. Pintelon R, Schoukens J (2001) System identification: a frequency domain approach. Wiley, New York

18. Halliday DM, Rosenberg JR, Amjad AM, Breeze P, Conway BA, Farmer SF (1995) A framework for the analysis of mixed time series/point process data - theory and application to the study of physiological tremor, single motor unit discharges and electromyograms. Prog Biophys Mol Biol 64:237-278. https://doi. org/10.1016/s0079-6107(96)00009-0

19. Bobet J, Norman RW (1990) Least-squares identification of the dynamic relation between the electromyogram and joint moment. J Biomech 23:1275-1276. https://doi.org/10.1016/00219290(90)90386-h

20. Cohen J (1988) Statistical power analysis for the behavioral sciences, vol 2. Lawrence Erlbaum Associates, Hillsdale

21. Johansson H, Sojka P (1991) Pathophysiological mechanisms involved in genesis and spread of muscular tension in occupational muscle pain and in chronic musculoskeletal pain 
syndromes: a hypothesis. Med Hypotheses 35:196-203. https:// doi.org/10.1016/0306-9877(91)90233-O

22. van Dieen JH, Flor H, Hodges PW (2017) Low-back pain patients learn to adapt motor behavior with adverse secondary consequences. Exerc Sport Sci Rev 45:223-229. https://doi. org/10.1249/JES.0000000000000121

23. Karayannis NV, Smeets RJ, van den Hoorn W, Hodges PW (2013) Fear of movement is related to trunk stiffness in low back pain. PLoS ONE 8:e67779. https://doi.org/10.1371/journal.pone.00677 79

24. van der Hulst M, Vollenbroek-Hutten MM, Schreurs KM, Rietman JS, Hermens HJ (2010) Relationships between coping strategies and lumbar muscle activity in subjects with chronic low back pain. Eur J Pain (London, England) 14:640-647. https://doi. org/10.1016/j.ejpain.2009.10.011

25. Osumi M, Sumitani M, Otake Y, Nishigami T, Mibu A, Nishi Y, Imai R, Sato G, Nagakura Y, Morioka S (2019) Kinesiophobia modulates lumbar movements in people with chronic low backpain: a kinematic analysis of lumbar bending and returning movement. Eur Spine J 28(7):1572-1578. https://doi.org/10.1007/ s00586-019-06010-4

26. van Dieen JH, Reeves NP, Kawchuk G, van Dillen LR, Hodges PW (2019) Motor control changes in low back pain: divergence in presentations and mechanisms. J Orthop Sports Phys Ther 49:370-379. https://doi.org/10.2519/jospt.2019.7917

27. Bourdon E, Graham RB, van Dieen JH (2019) A comparison of methods to quantify control of the spine. J of Biomech 96:1-7. https://doi.org/10.1016/j.jbiomech.2019.109344

28. Goodworth AD, Peterka RJ (2009) Contribution of Sensorimotor Integration to Spinal Stabilization in Humans. J Neurophysiol 102:496-512. https://doi.org/10.1152/jn.00118.2009

Publisher's Note Springer Nature remains neutral with regard to jurisdictional claims in published maps and institutional affiliations.

\section{Affiliations}

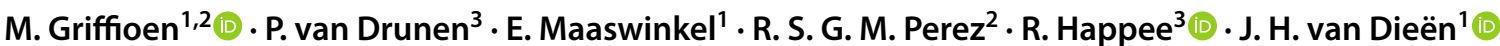

1 Department of Human Movement Sciences, Amsterdam Movement Sciences, Vrije Universiteit Amsterdam, Van der Boechorststraat 9, 1081 BT Amsterdam, The Netherlands

2 Department of Anesthesiology, VU University Medical Center Amsterdam, De Boelelaan 1117, 1081 HV Amsterdam, The Netherlands
3 Biomechanical Engineering Department, Faculty of Mechanical Engineering, Delft University of Technology, Mekelweg 4, 2628 CD Delft, The Netherlands 